\section{Quantitative biomarkers of stem cell differentiation based on intrinsic two-photon excited fluorescence}

William L. Rice, David L. Kaplan, and Irene Georgakoudi Tufts University, Biomedical Engineering Department, 4 Colby Street, Room 153, Medford, Massachusetts 02155

\begin{abstract}
We present the use of two-photon excited fluorescence (TPEF) as a noninvasive means to monitor differentiation of human mesenchymal stem cells (hMSCs) into an adipogenic pathway relying entirely on endogenous sources of contrast. Specifically, we demonstrate that TPEF can be used to reveal quantitative differences in the biochemical status and the shape of differentiating and nondifferentiating stem cells in two-dimensional (2-D) cultures. We find that even in simple 2-D cultures, not all cells are undergoing differentiation at the same rate. Last, such noninvasive approaches may also ultimately allow for determination of the lineage toward which the cells are differentiating (e.g., fat versus bone). Thus, intrinsic TPEF imaging provides quantitative morphological and biochemical biomarkers associated with stem cell differentiation and could serve as an important enabling technology in tissue engineering applications. () 2007 Society of PhotoOptical Instrumentation Engineers. [DOI: 10.1117/1.2823019]
\end{abstract}

Keywords: two-photon excited fluorescence (TPEF); tissue engineering; stem cells; adipogenic; differentiation; autofluorescence.

Paper 07234LRR received Jun. 30, 2007; revised manuscript received Oct. 5, 2007; accepted for publication Oct. 5, 2007; published online Dec. 18, 2007; corrected Feb. 29, 2008.

Tissue engineering approaches hold great promise for the development of functional tissue that can be used to improve, repair, or replace damaged tissues and organs. ${ }^{1}$ Most tissue engineering endeavors involve the placement of pluripotent cells, such as adult human mesenchymal stem cells (hMSCs), within a biomaterial scaffold and an environment that will provide a variety of chemical and or physical stimuli that ultimately induce cell differentiation and development of a particular tissue phenotype, such as bone, fat, heart, eye, etc. The timing of the stimuli can be as important to the outcome of the tissue as the nature of the stimulus itself. ${ }^{2}$ Thus, to enable efficient protocol optimization for these types of changes, it is important to be able to monitor the progress of the engineered tissue throughout the entire process. Of interest are cell proliferation, viability, metabolism, and morphology, as well as interactions with scaffolding and the extracellular matrix.

Currently, these parameters are monitored through invasive procedures that rely on serial biopsies or the use of multiple

Address all correspondence to Irene Georgakoudi, Tufts University, Biomedical Engineering Department, 4 Colby Str., Rm. 153, Medford, MA 02155. Tel: 617 627-4353; Fax: 617-627-3231; E-mail: Irene.Georgakoudi@tufts.edu experimental replicates that are sacrificed gradually at selective time-points. While these destructive assays yield a wealth of useful information, the maintenance of experimental culture replicates for monitoring the progression of the engineered tissue is costly, labor intensive, selective only for the time-points assessed, and at best an indirect measure of the primary sample. Therefore, noninvasive approaches could make a significant impact in this area. ${ }^{3}$

Optical technologies such as multiphoton microscopy (MPM) are generally considered noninvasive and have recently been investigated for monitoring engineered tissue components. ${ }^{4}$ Evaluation of cellular activity with optical microscopy is long established in the literature, through the intrinsic fluorescence of oxidized flavoproteins (FPs) and reduced pyridine nucleotide (NADH and NADPH) ${ }^{4,5}$ In this letter, we explore the use of two-photon excited fluorescence (TPEF) at 800-nm excitation as a noninvasive method for assessing biochemical and morphological cell features that can be correlated to adipogenic stem cell differentiation. At this wavelength, we expect that the observed fluorescence originates primarily from $\mathrm{NAD}(\mathrm{P}) \mathrm{H}$ (i.e., $\mathrm{NADH}$ and $\mathrm{NADPH}$ ), FPs, and retinol, while lipids and vitamin $\mathrm{D}$ are probably minor contributors. ${ }^{6}$ The inherent depth-resolution capabilities of this imaging modality should allow us to directly extend our findings to more complicated differentiation studies in three-dimensional (3-D) scaffolds. In this study, we monitor and compare the fluorescence of differentiating and nondifferentiating stem cells in the $455 \mathrm{~nm} \pm 35 \mathrm{~nm}$ and $525 \mathrm{~nm} \pm 25 \mathrm{~nm}$ regions of the spectrum over a period of three weeks. NADH fluorescence is expected to have major contributions in the 455-nm detector, flavins will be detected primarily by the $525-\mathrm{nm}$ one, while retinol can contribute significantly to the fluorescence acquired by both detectors. Using these images, we extract the fluorescence ratio, cell area, and eccentricity as quantitative biochemical and morphological biomarkers that we can correlate with differentiation status.

TPEF images were acquired on a Leica confocal microscope (IMDRE2) with a TCS-SP2 scan head and a Ti:Sapphire laser light source (Mai Tai, Spectra Physics) tuned to $800 \mathrm{~nm}$ with an average power at the sample of $8 \mathrm{~mW}$. The fluorescence was collected by two nondescanned PMTs through a filter cube with a 700-nm short-pass filter (HQ700sp 2p, Chroma) to reject the excitation light, a dichroic mirror (495dcxr, Chroma), a $455 \mathrm{~nm} \pm 35 \mathrm{~nm}$ bandpass filter (HQ455 70m2p, Chroma) and a $525 \mathrm{~nm} \pm 25 \mathrm{~nm}$ bandpass filter (HQ525 50m, Chroma). A six-frame average was acquired for each image. This level of exposure was found to yield on average only a $3 \%$ decrease in the mean intensity of an image for both detectors. The resulting images were analyzed with the MATLAB software package (Mathworks, Natick, Massachusetts). Specifically, the average fluorescence intensity from an area devoid of cells was measured and subtracted as background for the images from each detector. Cellular fluorescence was then measured as the mean intensity of all pixels within a manually selected region bounding the cell. Dark pixels were excluded such that the nucleus and lipid droplet areas did not contribute to the mean cellular fluorescence intensity estimates. The same regions were ana-

1083-3668/2007/12(6)/060504/3/\$25.00 @ 2007 SPIE 


\section{JBO LETTERS}
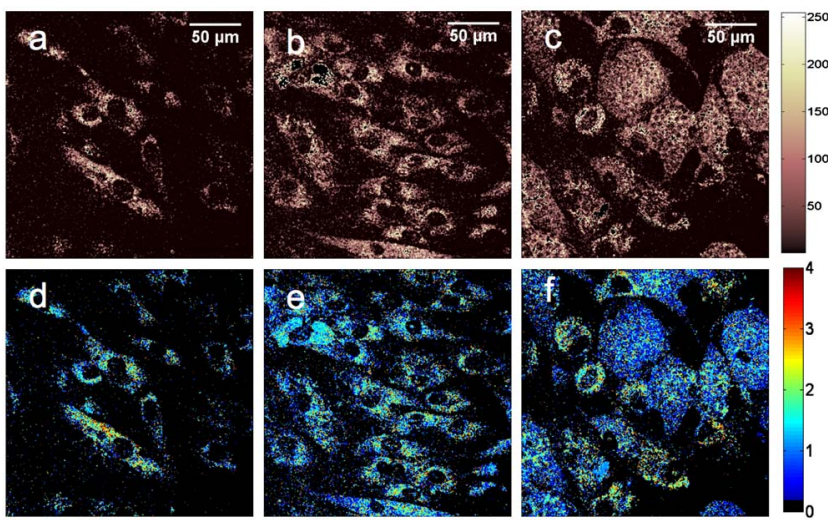

Fig. 1 Intrinsic fluorescence images of human mesenchymal stem cells exposed to adipogenic differentiation factors. The TPEF detected at $455 \mathrm{~nm}[(\mathrm{a}),(\mathrm{b}),(\mathrm{c})]$ and the resulting fluorescence ratio [(d), (e), (f)] images are shown on days $3[(\mathrm{a}),(\mathrm{d})], 8[(\mathrm{~b}),(\mathrm{e})$ and $21[(\mathrm{c}),(\mathrm{f})]$. Fluorescence ratio images $[(d),(e),(f)]$ reveal the changing cellular morphology and biochemical status of cells undergoing differentiation in false color, with blue likely indicating more metabolically active cells. The scale bar corresponds to $50 \mu \mathrm{m}$. (Color online only.)

lyzed in each corresponding image pair from the $455-\mathrm{nm}$ and 525-nm detectors.

Human mesenchymal stem cells (hMSCs) were isolated from adult bone marrow aspirates ${ }^{7}$ and maintained with previously described culture medium containing propagation or adipogenic differentiation factors. ${ }^{8}$ on culture dishes with coverslip bottoms (MatTek, Ashland, Massachusetts) for 21 days. At the end of the study, differentiation was confirmed by assessing the expression of the adipocyte marker lipoprotein lipase (LPL) and the osteocyte associated marker alkaline phosphatase (AP) using RT-PCR.

In Fig. 1, we show representative measured fluorescence images of hMSCs under differentiation conditions acquired with the 455-nm detector at early (day 3), mid (day 8), and late (day 21) time-points. The fluorescence ratio images, defined simply as the pixel-by-pixel ratio of the intensity detected by the $525-\mathrm{nm}$ detector to that of the $455-\mathrm{nm}$ detector, are displayed in false color, where red and yellow indicate a high ratio and blue represents a low ratio. We note that the computed fluorescence ratio images retain the morphological information of the measured fluorescence images, while providing visually accessible biochemical data. We detect significant differences in the levels of the fluorescence and in cell morphology as a function of incubation time in differentiation media. Specifically, cells induced into adipogenic phenotypes have accumulated a large number of lipid droplets, which can be seen as voids in the TPEF images, and have a larger, rounder appearance. We note also that the differentiating hMSCs display a lower fluorescence ratio than the cells at the onset of treatment.

The corresponding images of hMSCs cultured in propagation medium shown in Fig. 2, indicate that nondifferentiating cells maintain their elongated morphology over the 21-day period and exhibit a different profile of fluorescence intensity changes. Specifically, we observe that the fluorescence ratio of nondifferentiating hMSCs is maintained at fairly elevated values throughout treatment. Thus, the biochemical and mor-
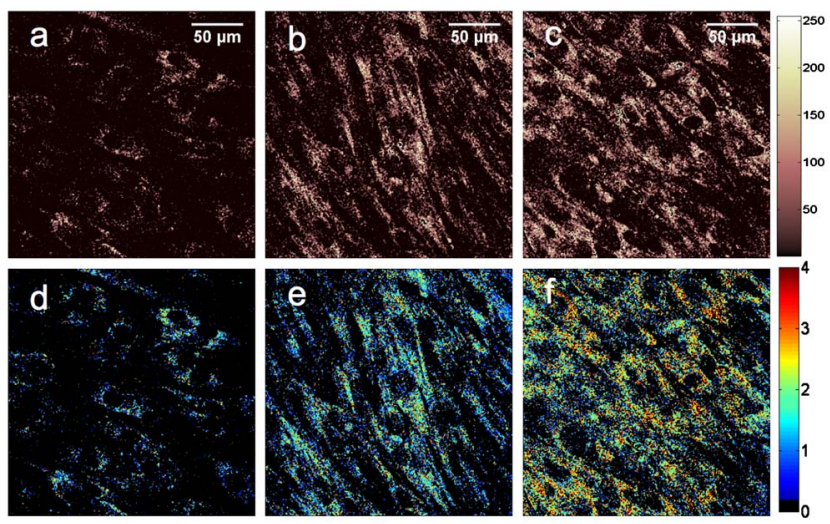

Fig. 2 Intrinsic fluorescence images of human mesenchymal stem cells exposed to propagation medium. The TPEF detected at $455 \mathrm{~nm}$ $[(a),(b),(c)]$ and the resulting fluorescence ratio $[(d),(e),(f)]$ images were aquired on days $2[(\mathrm{a}),(\mathrm{d})], 9[(\mathrm{~b}),(\mathrm{e})]$, and $21[(\mathrm{c}),(\mathrm{f})]$. Fluorescence ratio images $[(d),(e),(f)]$ reveal the maintenance of elongated cellular morphology and biochemical state of cells in propagation medium in false color, with red and yellow likely indicating a low level of metabolic activity. The scale bar is $50 \mu \mathrm{m}$. (Color online only.)

phological changes noted in Fig. 1 are attributed to stem cell differentiation.

These observations are represented in a more quantitative fashion in Fig. 3, based on measurements of typically 15 to 20 cells visible in two microscopic fields of a culture dish at each time-point. Specifically, we find that the fluorescence intensity detected at $455 \mathrm{~nm}$ and $525 \mathrm{~nm}$ increases as a function of time for both groups of cells. However, the rate of increase in the blue fluorescence intensity $(455 \pm 35 \mathrm{~nm})$ is higher for the differentiating cells, with a statistically significant increase $(\mathrm{p}<0.01$, based on a two-tailed student's t-test) noted on days 14 and 17 of differentiation treatment compared to cells in propagation medium. The levels of green/yellow fluorescence $(525 \pm 25 \mathrm{~nm})$ is similar for the two groups up to the $17 \mathrm{th}$ day of treatment. However, the mean intensity per cell measured at this wavelength regime becomes significantly lower $(p<0.01)$ on the 21 st day of culture for the differentiating than for the nondifferentiating cells.

The mean fluorescence ratio for each of the cells included in these calculations is shown in Fig. 3(c). We note that initially, the range of fluorescence ratios is similar for the stem cells in the propagation and differentiation groups, as expected. However, as incubation time increases, we observe that the fluorescence ratio of cells in the differentiation medium shifts to values lower than that of cells in propagation medium. In fact, the mean fluorescence ratio for differentiating stem cells is statistically lower $(p<0.01)$ than that for the cells in propagation medium for all measurements conducted on days 8 to 21 . Fluorescence ratio values are included in Fig. 3 (c) as a function of the product of two morphological parameters that we acquire from the TPEF cell images. Specifically, we multiply the area of the cell (in thousands of pixels) with a factor indicating how circular the cells are. This factor is defined as 1 minus the eccentricity (measured as the ratio of the distance between the foci of the major and minor axies of an ellipse encompassing the cell) and is thus equal to 0 for a line and 1 for a perfect circle. These factors are used to rep- 


\section{JBO LETTERS}
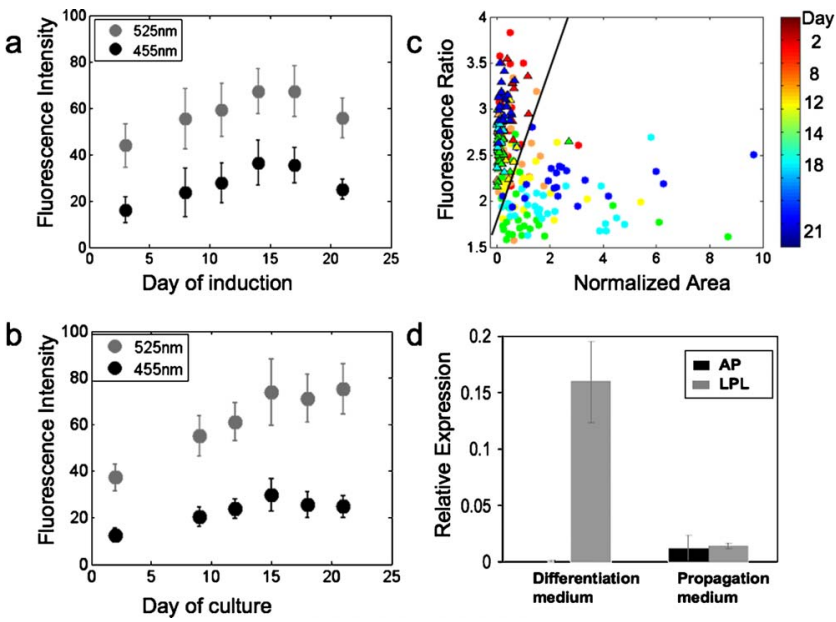

Fig. 3 Quantitative analysis of differentiating and nondifferentiating stem cells. The mean intensity per cell of TPEF from the $455 \mathrm{~nm}$ (black) and $525 \mathrm{~nm}$ (gray) detectors is reported over 21 days in cells undergoing differentiation (a) and those maintained undifferentiated in propagation medium (b). (c) The fluorescence ratio and the morphology normalized area of the cells can be used as quantitative biomarkers to separate nondifferentiating cells (filled triangles) from an increasing number of cells in differentiation medium (filled circles), as suggested from the line drawn based on visual data inspection. Different colors represent progressive days of exposure to propagation or differentiation medium. (d) RT-PCR confirms strong expression of lipoprotein lipase (LPL), an adipogenic differentiation marker, and lack of expression of alkaline phosphate (AP), an osteogenic marker, in cells incubated with adipogenic differentiation medium. Stem cells maintained in propagation medium do not express LPL or AP. (Color online only.)

resent quantitatively a significant increase in the area and the circularity of differentiating cells. It is interesting to note based on the results in Fig. 1 and Fig. 3(c) that not all cells are undergoing adipogenic differentiation at the same rate, even in simplified two-dimensional (2-D) cultures. This is important, since most molecular characterization techniques assess global changes. In addition, we note that it is possible to draw a simple line based on visual inspection of the data, to separate all but 1 out of the 117 cells in propagation medium from $10 \%, 23 \%, 70 \%, 77 \%, 96 \%$, and $90 \%$ of the cells in differentiation medium imaged on days $2,8,12,14,18$, and 21 of treatment, respectively. Adipogenic differentiation is confirmed by RT-PCR analysis [Fig. 3(d)]. LPL, a marker of adipogenic differentiation, is expressed in hMSCs maintained in adipogenic differentiation medium while expression of AP is not detected. Only low levels of LPL and AP are expressed in stem cells maintained in propagation medium.

The increase of blue fluorescence seen in differentiating cells relative to those in propagation medium could be attrib- uted to an increase in NADH. Considering that NADH fluorescence also contributes to green/yellow fluorescence, the lack of a significant increase in the fluorescence detected at $525 \mathrm{~nm}$ from differentiating cells may be due to a decrease in FPs. This complementary increase of NADH and decrease of FPs would be consistent with an overall increase in metabolic activity of differentiating cells. Further studies performed at additional excitation wavelengths are necessary to identify the origins of such changes more accurately.

In summary, this initial study demonstrates the potential of intrinsic TPEF imaging to provide quantitative biochemical and morphological biomarkers that can be used to monitor stem cell differentiation into an adipogenic pathway. We expect that this modality may also be sensitive to changes that are associated with other differentiation pathways, such as those involved in bone, cartilage, and vascular tissue development, as well as provide a means for monitoring cell/matrix interactions in engineered tissues.

\section{Acknowledgments}

We would like to acknowledge Joshua Mauney for help with stem cell differentiation protocols. The study was supported by NSF Grant No. BES0547292 and NIH Grant No. P41EB002520.

\section{References}

1. J. P. Vacanti and R. Langer, "Tissue engineering: the design and fabrication of living replacement devices for surgical reconstruction and transplantation," Lancet 354 Suppl 1, SI32-4 (1999).

2. G. F. Muschler, C. Nakamoto, and L. G. Griffith, "Engineering principles of clinical cell-based tissue engineering," J. Bone Jt. Surg., Am. Vol. 86-A(7), 1541-1558 (2004).

3. J. J. Pancrazio, F. Wang, and C. A. Kelley, "Enabling tools for tissue engineering," Biosens. Bioelectron. (2006).

4. K. Schenke-Layland, I. Riemann, O. Damour, U. A. Stock, and K. Konig, "Two-photon microscopes and in vivo multiphoton tomographs-powerful diagnostic tools for tissue engineering and drug delivery," Adv. Drug Delivery Rev. 58(7), 878-896 (2006).

5. A. Shiino, M. Haida, B. Beauvoit, and B. Chance, "Threedimensional redox image of the normal gerbil brain," Neuroscience 91(4), 1581-1585 (1999).

6. W. R. Zipfel, R. M. Williams, R. Christie, A. Y. Nikitin, B. T. Hyman, and W. W. Webb, "Live tissue intrinsic emission microscopy using multiphoton-excited native fluorescence and second harmonic generation," Proc. Natl. Acad. Sci. U.S.A. 100(12), 7075-7080 (2003).

7. L. Meinel, V. Karageorgiou, S. Hofmann, R. Fajardo, B. Snyder, C. Li, L. Zichner, R. Langer, G. Vunjak-Novakovic, and D. L. Kaplan, "Engineering bone-like tissue in vitro using human bone marrow stem cells and silk scaffolds," J. Biomed. Mater. Res. 71(1), 25-34 (2004).

8. J. R. Mauney, V. Volloch, and D. L. Kaplan, "Matrix-mediated retention of adipogenic differentiation potential by human adult bone marrow-derived mesenchymal stem cells during ex vivo expansion," Biomaterials 26(31), 6167-6175 (2005). 